\title{
EVALUACIÒN DE LOS FACTORES NUTRICIONALES Y ANTINUTRICIONALES DE CUATRO ESPECIES VEGETALES QUE PUEDEN TENER POTENCIAL FORRAJERO
}

\author{
Fonseca $\mathrm{M}, \mathrm{A}^{1}$, Barreto, $\mathrm{L}^{2}$, Granados, $\mathrm{J}^{3}$ \\ ${ }^{1}$ Tesista programa de zootecnia \\ ${ }^{2}$ Zootecnista, Esp. Nutrición Animal, MSc, Desarrollo Rural, PhD Desarrollo Sostenible \\ ${ }^{3}$ Lic. Químico. Magister en Docencia de la Química. MSc
}

\section{RESUMEN}

Propendiendo por el fomento de la producción agropecuaria con criterio sustentable y sostenible y dentro del marco ecológico se seleccionaron cuatro especies vegetales propias de las regiones del trópico alto de la cordillera oriental colombiana, Se analizaron diferentes factores nutricionales y antinutricionales, así como el índice de digestibilidad in vitro. Se determinó el análisis químico proximal (AQP) de materia seca (MS) materia orgánica (MO), materia inorgánica (cenizas, CZ) y proteína cruda (PC). Mediante las variables fraccionales de Van Soest se analizaron los porcentajes de proteína verdadera (PV), fibra detergente neutra (FDN) y fibra detergente àcida (FDA); por el método in vitro de Tilley y Terry se determinó el porcentaje de digestibilidad de la proteína, y se determinaron polifenoles totales (PFT), y cuantitativa y cualitativamente otros factores antinutricionales (FAN). Los rangos dentro de los cuales se determinaron los porcentajes de las variables son: MS 35,86 - 39,12; MO 92,33 - 98,24; CZ 1,76 - 7,67; PC 15,88 - 25,58; PV 15,62 -25,19; FDN 31,51 - 63,16; FDA 21,8 - 40,95; Digestibilidad 28,51-43,75; PFT 3,56 - 21,16; taninos hidrosolubles (TH) 1,61 - 6,81; igualmente desde el punto de vista cualitativo presentaron de poco a moderado contenido de saponinas, presencia moderada a alto contenido de terpenos saturados, presentaron fenoles catecoles, algunos esteroides y poca presencia de alcaloides. El modelo estadístico experimental que se aplicó fue completamente al azar, el cual se define como: un procedimiento físico experimental, aplicando medios que aseguran que cada tratamiento tiene una probabilidad igual de ser puesto a prueba sobre cualquier grupo particular de unidades experimentales (Fisher, 1935). Los datos obtenidos en el trabajo de laboratorio fueron analizados usando el programa para Windows SPSS 15.0; con dos factores, (a) zonas (fototrópica, media y geotrópica); (b) corresponde a cuatro especies. Modelo para el factor $\mathrm{a} * \mathrm{~b}$ con tres réplicas totalmente al azar para cada zona.

Palabras clave: Nutricional, Digestibilidad, Antinutricional, Potencial forrajero

DOI: https://doi.org/10.22490/26653176.2342 


\section{SUMMARY}

Strive for the promotion of sustainable agricultural production and sustainable judiciously and within the ecological framework, in four plant species typical of tropical regions of the eastern mountains high in Colombia, were analyzed various nutritional and antinutritional factors and the rate of digestibility in vitro. We determined the proximate analysis (AQP) of dry matter (DM) and organic matter $(\mathrm{OM})$, inorganic matter (ash, $\mathrm{CZ}$ ) and crude protein $(\mathrm{CP})$; by the Van Soest fractional variables were analyzed for true protein percentages ( PV), neutral detergent fiber (NDF) and acid detergent fiber (FDA), for the in vitro method of Tilley and Terry is determined the percentage of protein digestibility and total polyphenols were determined (PFT), and other quantitative and qualitative antinutritional factors (ANF). The ranges within which to determine the percentages of the variables are: MS 35.86 to 39.12 , MO 92.33 to 98.24 ; CZ 1.76 to 7.67 ; PC 15.88 to 25.58 ; PV 15.62 to 25.19, NDF 31.51 to 63.16 ; FDA from 21.8 to 40.95 ; digestibility from 28.51 to 43.75 ; PFT3.56 to 21.16; soluble tannins (TH ) from 1.61 to 6.81 , also from the qualitative point of view had little to moderate content of saponins, the presence of moderate to high in saturated terpenes, phenols, catechols had some little presence of steroids and alkaloids. The experimental statistical model was used, completely random, which is defined as an experimental physical process, using resources that ensure that each treatment has an equal probability of being tested on any particular group of experimental units (Fisher, 1935). The data obtained in the laboratory work were analyzed using SPSS 15.0 for Windows program, with two factors, (a) areas (phototropic, medium and geotropic), (b) corresponds to four species. Model for the factor $\mathrm{a} * \mathrm{~b}$ with three replicates, totally random.

Keywords: Nutricional, Antinutritional. Digestibility, Forage potential

\section{INTRODUCCIÒN}

Nuevas alternativas en la producción, aplicadas en la nutrición animal han propiciado la selección de algunas plantas, se han analizado varios aspectos de la forma sustentable y sostenible de la productibilidad agropecuaria por lo tanto se han realizado estudios en laboratorio de la caracterización bromatológica con el propósito de determinar el uso no solo como plantas que ecológicamente participan activamente en el equilibrio de un entorno ambiental, sino que podrían ser usadas como forraje básico o complementario en la alimentación de animales de granja.

En este sentido en un trabajo reportado por Cárdenas, 2006, hace una descripción de varias especies forrajeras que predominan en las regiones de trópico alto colombiano y que en el aspecto silvoagricola pueden ser alternativa para ser utilizadas como forraje. En otros países igualmente se está trabajando en este sentido, al respecto Carranza, et al, 2003, presentaron un trabajo que determinó diecinueve especies entre herbáceas, arbustivas y arbóreas del bosque tropical caducidófilo de México de las cuales evaluaron la producción de biomasa y 
análisis bromatológico. Por otra parte, Botero, et al, 2002, presentan un trabajo orientado en el estudio de la utilización en sistemas sostenibles de producción animal de árboles y arbustos fijadores de nitrógeno en suelos ácidos tropicales. García, et al, 2009, publicaron un trabajo en el cual reportan la caracterización de forrajes leguminosos y otras especies desde el punto de vista químico-nutricional. Por su parte Suarez, et al, 2008, presentaron los resultados de la investigación sobre su composición química y la digestibilidad in vitro de algunas especies arbóreas del piedemonte Amazónico. En Brasil, de Araujo, et al, 2002, presentaron el estudio del contenido de nutrientes y de la digestibilidad in vitro de la materia seca del follaje de algunos árboles forrajeros de la Catinga. En el medio tropical (Barahona $\mathrm{R}$, et al; 2005) analizaron la digestibilidad in vitro promedio de la proteína, determinando las limitaciones causadas en la productividad animal causada por la fibra detergente neutra, contenida en los forrajes. Santacoloma, et al, 2010, evalúan el contenido de metabolitos secundarios en dos especies de plantas forrajeras encontradas en dos pisos térmicos de Colombia. Finalmente, Santacoloma, 2011, afirma que hay que tener presente, que muchos forrajes de baja digestibilidad y valor nutricional, regularmente contienen metabolitos secundarios que reducen tanto la población de protozoarios en el rumen como la de bacterias metanogénicas, lo cual posibilita una disminución de la emisión de metano a la atmósfera y mejora los patrones de fermentación ruminal.

El propósito fundamental de este trabajo es analizar los diferentes factores nutricionales y antinutricionales, así como el índice de digestibilidad in vitro de cuatro especies arbustivas del trapico alto de la cordillera oriental. El resultado final de este estudio puede determinar la aplicación apropiada de las plantas estudiadas; aunque si bien el objetivo primordial es buscar alternativas nutricionales, es decir, como forraje para animales, también podrían ser útiles y cumplir otras funciones agroecológicas.

\section{OBJETIVOS}

Seleccionar cuatro especies vegetales arbustivas; realizar la caracterización en el laboratorio de nutrición de sus factores fisicoquímicos, nutricionales, antinutricionales, digestibilidad in vitro de la proteína, según las técnicas respectivas de Weende, Van Soest, Kjeldahl, Tilley y Terry. Con base en los resultados obtenidos, realizar la evaluación cuantitativa y para algunas variables la evaluación cualitativa de cada uno de los factores analizados y calificados. La evaluación para cada especie permite en la práctica determinar las condiciones propias para ser tenida en cuenta como forraje; no implica sin embargo que la especie no pueda aportar otras propiedades ecológico-ambientales en una unidad productiva agropecuaria.

\section{OBJETIVOS ESPECÍFICOS}


- Determinar por el método fisicoquímico el área foliar (AF), clorofilas, materia seca (MS), materia inorgánica $(\mathrm{Cz})$ y por el método potenciomètrico el $\mathrm{pH}$.

- Analizar por la técnica analítica de Kjeldahl el porcentaje de nitrógeno total (NT), proteína cruda (PC), nitrógeno no proteico (NNP) y proteína no verdadera (PNV).

- Establecer el porcentaje, mediante el análisis proximal o de Weende la proteína total (PT), fibra detergente neutra (FDN), fibra detergente ácida (FDA), lignina detergente àcida (LDA), nitrógeno no proteico (NNP).

- Conocer la digestibilidad de la proteína mediante el método in-vitro de pepsina- pancreatina de Terry y Tilley.

- Evaluar mediante el método espectrofotométrico los factores antinutricionales (FAN).

\section{METODOLOGÌA}

En el ámbito del desarrollo sostenible y sustentable, con este trabajo de investigación realizado en el laboratorio de nutrición animal de la UNAD, Cead, Josè Celestino Mutis en Bogotá; se desarrolló la caracterización desde el punto de vista nutricional y antinutricional, de cuatro especies vegetales pertenecientes al trópico alto de la cordillera oriental colombiana. Se tomaron tallos y hojas tiernas, de plantas maduras sembradas en estado natural, es decir, en un ambiente muy poco alterado por la presencia humana.

Las plantas seleccionadas fueron la Fucsia (Fuchsia boliviana), Pajarito (Crotalaria agatiflora), Roble amarillo (Tecoma stans) y Encenillo (Weinmannia tomentosa). Teniendo en cuenta las características generales de cada planta en los aspectos tanto de las condiciones ambientales en que se encontraron, el suelo, la humedad, el estado sanitario y estado vegetativo, se realizó muestreo de cada especie tres plantas, en tres niveles (fototrópico, medio y geotrópico); se homogeneizaron las muestras de cada planta según cada nivel para efecto de realizar el análisis de laboratorio, los porcentajes se promediaron para el análisis de varianza ANOVA acoplado a la prueba de rango múltiple de «Duncan» y test de Tukey; en una sola vìa con dos factores, (a) zonas (fototrópica, media y geotrópica); (b) corresponde a cuatro especies, modelo para el factor a * b con tres réplicas, totalmente al azar. Se realizó este análisis con el software SPSS 11 para Windows, contrastando los datos entre especies y entre zonas (fototrópico, medio, geotrópico), determinando un $95 \%$ y $99 \%$ de confiabilidad. La ecuación se presenta así:

$$
\text { Yijk }=\mu+\mathbf{T i}+\square \mathbf{j}+\mathbf{T} \square \mathbf{i j}+E \mathbf{i j k}
$$

Donde: Yijk: Valores de las variables analizadas, observadas en la unidad experimental 
(ijk); $\boldsymbol{\mu}$ : Media; Ti: Efecto zonas; $\square \mathbf{j}$ : Efecto especies $(\mathbf{j}=1,2,3,4)$; Eijk: Error experimental; i: 1, 2, 3, (zonas lumínicas: fototrópica, media, geotrópica); j: 1, 2,3,4,

(especies estudiadas); $\mathbf{k}:$ 1, 2, 3, (réplicas $=1$ ).

\section{Métodos}

Los análisis en el laboratorio se han desarrollado de acuerdo con los respectivos protocolos determinados para este efecto, se dividieron en análisis fisicoquímicos, análisis quìmico proximal, variables fraccionales de Van Soest, digestibilidad in-vitro de proteína por el método Tilley y Terry, y los factores antinutricionales, de los cuales algunos se cuantificaron y otros se cualificaron según se describe más adelante.

La variable fisicoquímica del $\mathrm{pH}$ se determinó mediante el método potenciomètrico; el porcentaje de materia seca (MS) se determinó por el método de la diferencia de peso según el estado de humedad del material analizado hasta llegar a la calcinación total en el cual solo queda la materia inorgánica (minerales). Para determinar los porcentajes de nitrógeno total (NT), proteína cruda (PC), fibra detergente neutra (FDN), fibra detergente ácida (FDA), lignina detergente ácida (LDA), nitrógeno no proteico (NNP) se utilizó el método de Kjeldahl.

Por el método espectrofotométrico se han calculado los porcentajes de polifenoles totales (PFT) método colorimétrico de Folìn-Denis, nitrógeno soluble (NS); el porcentaje de la digestibilidad in vitro se realizó mediante el método de digestibilidad de proteína en pepsina-pancreatina (DIVPPP).

El proceso de experimentación en el laboratorio y las tècnicas para la realización de los análisis respectivos se reportan en la siguiente tabla:

Tabla 1: Resumen de variables analizadas, técnica utilizada y unidades presentadas

\begin{tabular}{llc}
\hline Variable evaluada & Tipo de variable & Unidades \\
\hline pH & Fisicoquímica & $\%$ \\
Materia seca & Análisis químico proximal & $\%$ \\
Materia orgànica & Análisis químico proximal & $\%$ \\
Cenizas & Análisis químico proximal & $\%$ \\
Proteìna cruda & Anàlisis quìmico proximal & $\%$
\end{tabular}




\begin{tabular}{llc} 
Nitrògeno no protèico & Fraccionales de Van Soest & $\%$ \\
Proteína verdadera & Fraccionales de Van Soest & $\%$ \\
Proteína no verdadera & Fraccionales de Van Soest & $\%$ \\
Fibra detergente neutra & Fraccionales de Van Soest & $\%$ \\
Fibra detergente àcida & Fraccionales de Van Soest & $\%$ \\
Lignina detergente àcida & Fraccionales de Van Soest & $\%$ \\
Hemicelulosa & Fraccionales de Van Soest & $\%$ \\
Digestibilidad & Método Tilley y Terry & $\%$ \\
Fitometabolitos & Factores antinutricionales & $\begin{array}{c}\text { Cuantitativos y } \\
\text { cualitativos }\end{array}$ \\
\hline
\end{tabular}

Todas las técnicas analíticas se han tomado de la AOAC, Van Soest.

AOAC (The Scientific Association Dedicated to Excellence in Analytical Methods)

En las tablas siguientes se reportan los porcentajes de las diferentes variables evaluadas en el orden en que corresponde a la técnica utilizada.

Tabla 2: Variable evaluada mediante la técnica fisicoquímica.

\begin{tabular}{lc}
\hline ESPECIES & $\mathbf{p H}$ \\
\hline Fuchsia boliviana & 5,84 \\
Crotalaria agatiflora & 6,38 \\
Tecoma stans & 6,23 \\
$\underline{\text { Weinmannia tomentosa }}$ & $\underline{6,2}$ \\
\hline
\end{tabular}

Se observa que los porcentajes del potencial de hidrógenos son muy similares entre las especies, no obstante tiende hacia la acidez la Fuchsia boliviana. En el gráfico 1 se aprecia el contraste en el potencial de hidrógenos $(\mathrm{pH})$ entre las tres zonas analizadas 


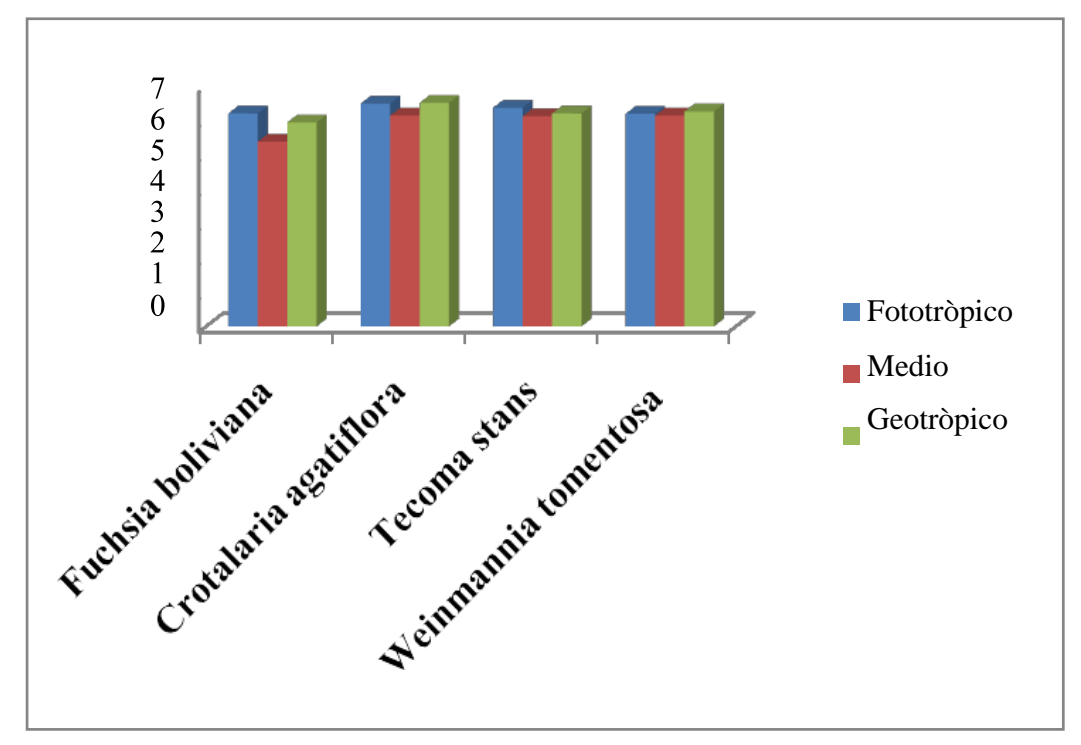

Gráfico 1: Contraste de $\mathrm{pH}$ entre zonas.

Tabla 3: Variables evaluadas mediante la técnica del análisis químico proximal.

\begin{tabular}{lccrr}
\hline ESPECIES & MS\% & Cz\% & MO\% & \multicolumn{2}{c}{ PC\% } \\
\hline Fuchsia boliviana & 36,36 & 2,99 & 97,14 & 19,14 \\
Crotalaria agatiflora & 38,81 & 1,76 & 98,24 & 25,59 \\
Tecoma stans & 37,42 & 7,67 & 92,33 & 15,89 \\
Weinmannia tomentosa & 39,02 & 2,71 & 97,29 & 16,29 \\
\hline
\end{tabular}

MS: Materia seca; Cz: Cenizas; MO: Materia orgánica; PC: Proteìna cruda.

Las variables MS y MO presentan cierta homogeneidad en los porcentajes hallados entre las especies, en la variable de la materia inorgánica (cenizas, $\mathrm{Cz}$ ) la Crotalaria agatiflora presentó el mayor porcentaje de $\mathrm{MO}$ y el menor porcentaje de $\mathrm{Cz}$, por el contrario la Tecoma stans reportó el menor porcentaje de $\mathrm{MO}$ y el mayor porcentaje de $\mathrm{Cz}$, estos datos permiten hacer la hipótesis de que el contenido de minerales es mayor en esta última especie dentro del porcentaje total de MS. 


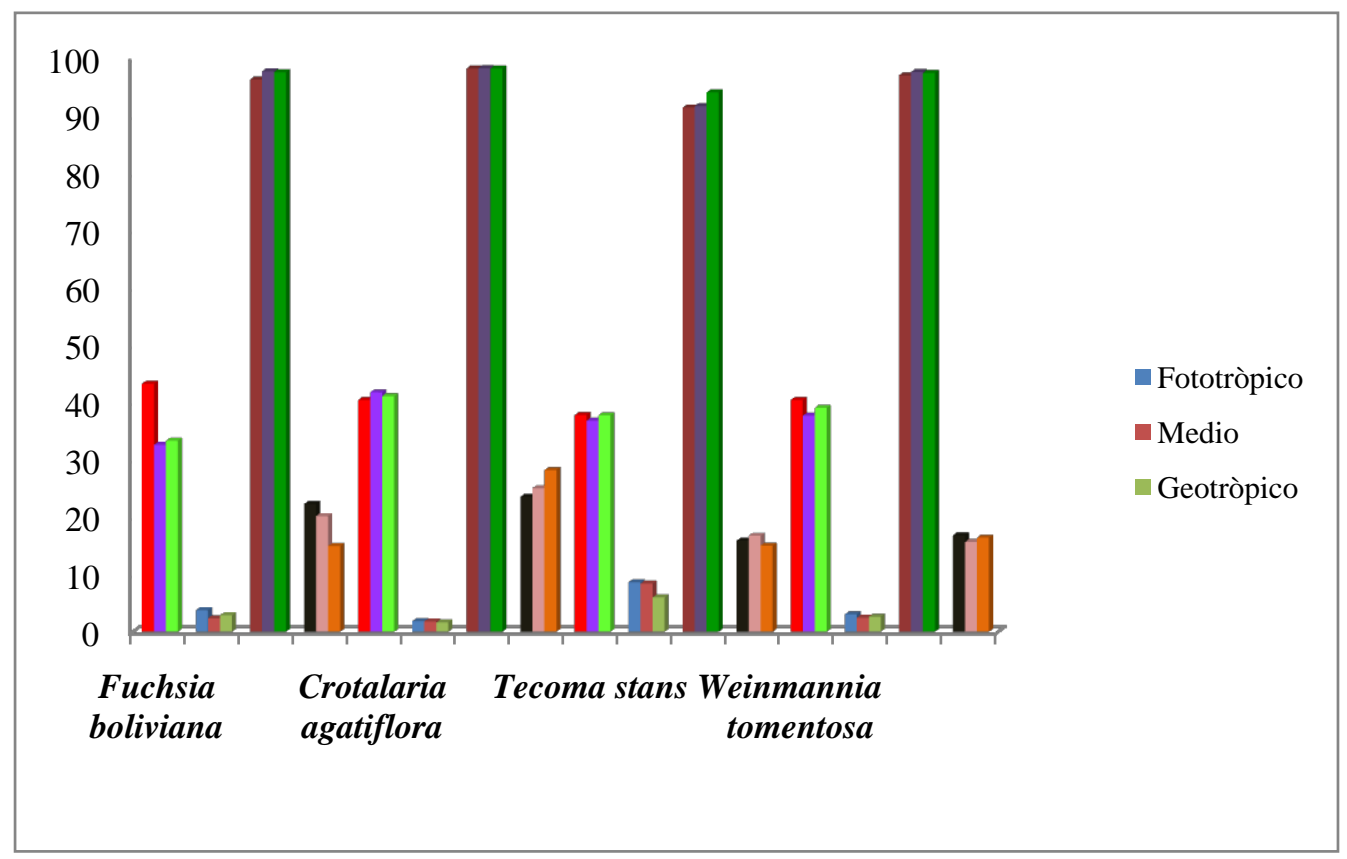

Gráfico 2: Contraste en su orden de MS; Cz; MO; PC entre zonas de cxada especie analizada.

Tabla 4: Variables evaluadas mediante la técnica del análisis fraccional de Van Soest.

\begin{tabular}{lccccccc}
\hline ESPECIES & NNP\% & PV\% & $\begin{array}{r}\text { PNV } \\
\%\end{array}$ & FDN\% & $\begin{array}{r}\text { FDA } \\
\%\end{array}$ & LDA\% & Hc\% \\
\hline Fuchsia boliviana & 0,18 & 18,04 & 1,09 & 31,51 & 21,8 & 14,62 & 9,72 \\
$\begin{array}{l}\text { Crotalaria agatiflora } \\
\text { Tecoma stans }\end{array}$ & 0,06 & 25,19 & 0,68 & 39,35 & 20,24 & 12,07 & 19,11 \\
$\begin{array}{l}\text { Weinmannia } \\
\text { tomentosa }\end{array}$ & 0,04 & 15,63 & 0,26 & 63,16 & 40,95 & 28,18 & 22,2 \\
& 0,07 & 15,87 & 0,42 & 41,46 & 39,68 & 23,6 & 1,78 \\
\hline
\end{tabular}

NNP: Nitrógeno no proteico; PV: Proteína verdadera; PNV: Proteína no verdadera;

FDN: Fibra detergente neutra; FDA: Fibra detergente ácida; LDA: Lignina detergente ácido; Hc: Hemicelulosa. 
En estos datos se observa que la hemicelulosa $(\mathrm{Hc})$, molècula que se une a otras moléculas mediante enlaces que constituyen la pared rígida que protege a la célula de la presión ejercida sobre ésta por el resto de las células que la rodean, en la especie Tecoma stans el porcentaje mas alto corresponde a este factor, que de acuerdo con los porcentaje que presenta la FDN, FDA, y la LDA para esta especie presentados en la tabla 3, afirma que según la hipótesis presentada, esta especie contiene el mayor porcentaje de materia inorgánica $(\mathrm{Cz})$.

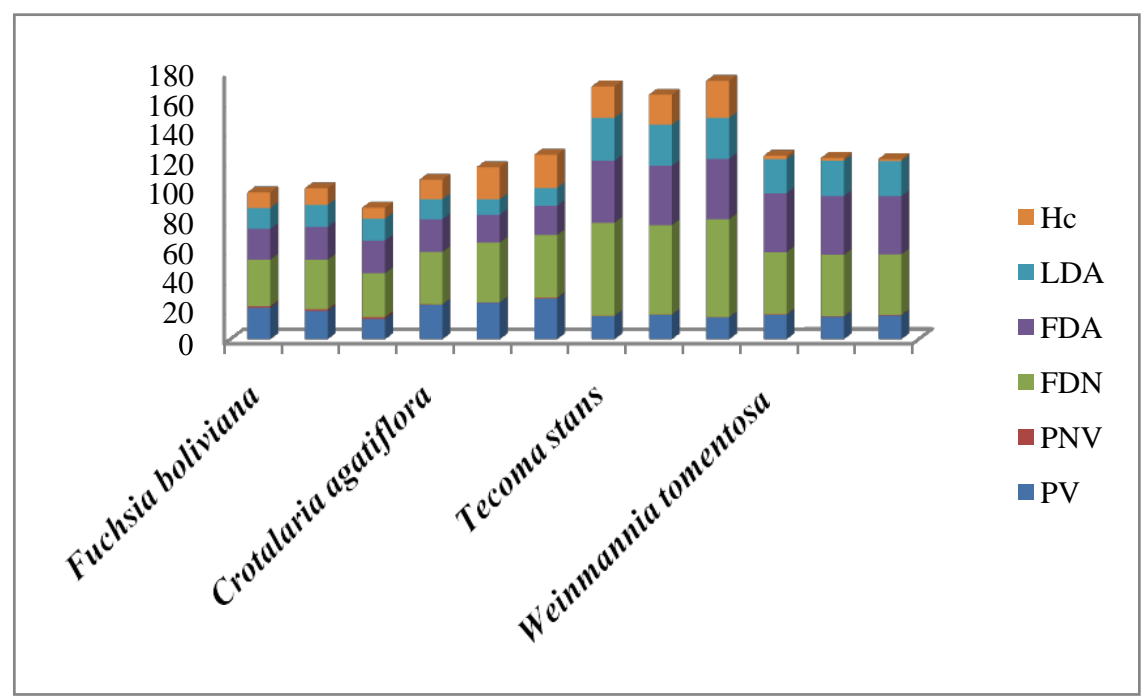

Gráfico 3: Contraste de las variables reportadas por zonas entre especies.

Tabla 5: Evaluación de la digestibilidad invitro por el método de Metodo Tilley y Terry.

\begin{tabular}{lr}
\hline ESPECIES & DIGEST \% \\
\hline Fuchsia boliviana & 28,51 \\
Crotalaria agatiflora & 28,9 \\
Tecoma stans & 43,75 \\
Weinmannia tomentosa & $\underline{43,03}$ \\
\hline
\end{tabular}




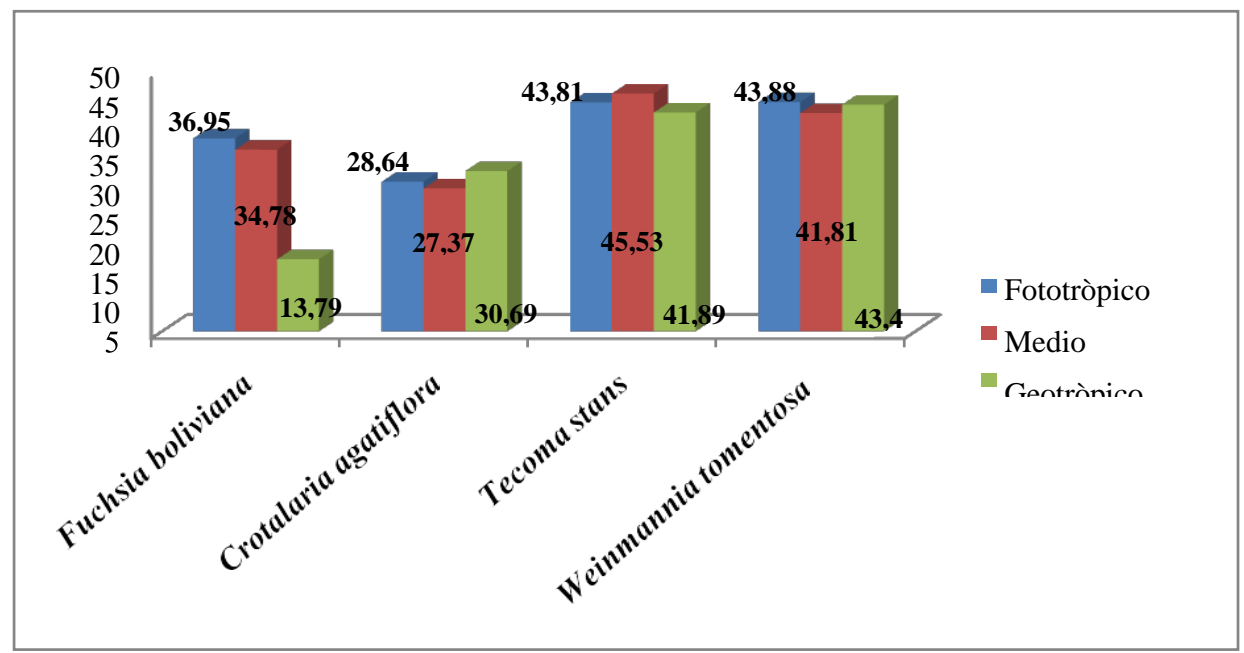

Gráfico 4: Contraste entre zonas de la variable digestibilidad.

De acuerdo con los porcentajes de digestibilidad in vitro reportados, presenta el mayor índice la Tecoma stans y la Weinmannia tomentosa, es posible hacer la hipótesis, teniendo en cuenta los porcentajes de FDN, FDA, LDA, que el contenido de estos factores puede ser altamente soluble, por lo tanto, bien aprovechable en términos prácticos en la alimentación animal.

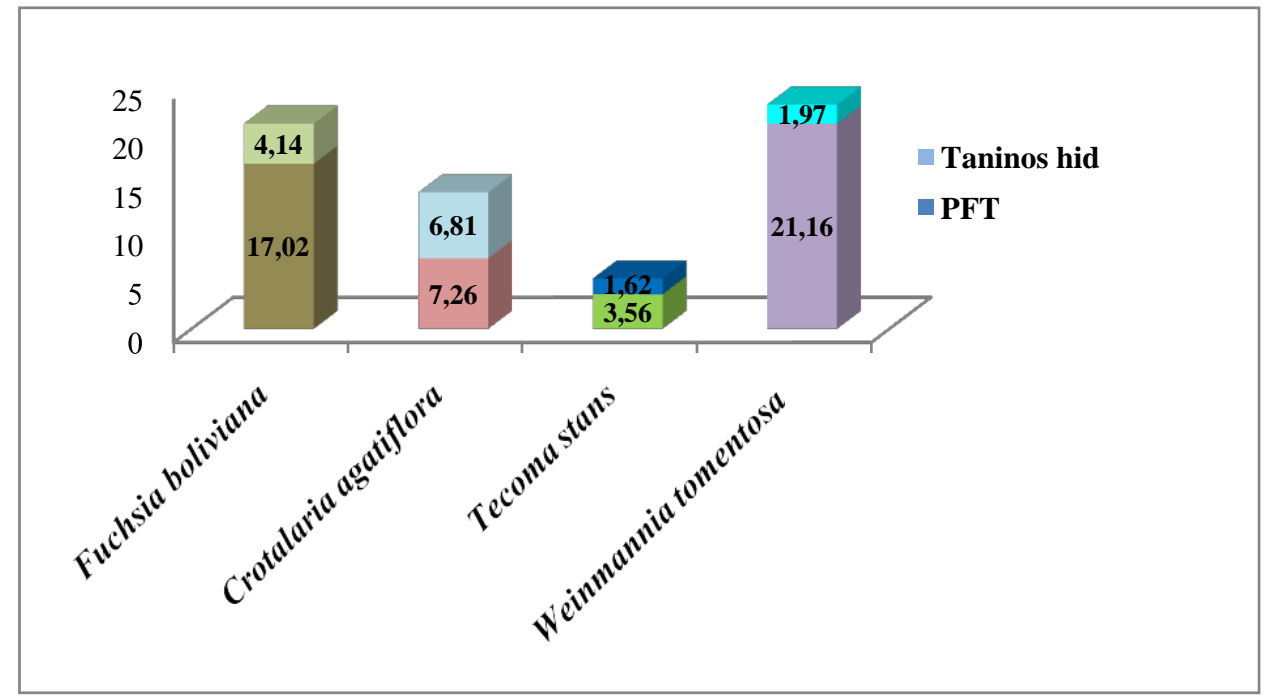

Gráfico 5: Evaluación de Fitometabolitos secundarios como factores antinutricionales (FAN) 
Los polifenoles son sustancias químicas compuestas por más de un grupo fenol por molécula. Se subdividen entre otros compuestos en lignina, taninos hidrolizables y taninos condensados. En el gráfico se aprecia la relación porcentual entre los PFT y los taninos hidrosolubles. Se realizó el análisis de algunos FAN, los cuales se presentan en la tabla siguiente.

Tabla 6: Evaluación cualitativa de factores antinutricionales (FAN)

\begin{tabular}{lcccccc}
\hline ESPECIES & Saponinas & $\begin{array}{c}\text { Fenoles } \\
\text { pirogálicos }\end{array}$ & $\begin{array}{c}\text { Terpenos } \\
\text { saturados }\end{array}$ & catecoles & Esteroides & Alcaloides \\
\hline $\begin{array}{l}\text { Fuchsia } \\
\text { boliviana }\end{array}$ & $\mathrm{N}$ & $* * *$ & $* *$ & $\mathrm{~N}$ & $\mathrm{~N}$ & $\mathrm{~N}$ \\
$\begin{array}{l}\text { Crotalaria } \\
\text { agatiflora }\end{array}$ & $\mathrm{N}$ & $* * *$ & $\mathrm{~N}$ & $\mathrm{~N}$ & $\mathrm{~N}$ & $*$ \\
$\begin{array}{l}\text { Tecoma stans } \\
\text { Weinmannia }\end{array}$ & $\mathrm{N}$ & $*$ & $*$ & $* *$ & $*$ & $*$ \\
tomentosa & $*$ & $*$ & $\mathrm{~N}$ & $\mathrm{~N}$ & $\mathrm{~N}$ & $*$ \\
\hline
\end{tabular}

$(\mathrm{N})$ : Negativo; $(*)$ : Muy baja presencia; $(* *)$ : Presencia; $(* * *)$ : Alta presencia

\section{Análisis estadístico}

El modelo estadístico experimental que se aplicó fuè completamente al azar, el cual se define como un procedimiento físico experimental, usando medios que aseguren que cada tratamiento tiene una probabilidad igual de ser puesto a prueba, sobre cualquier grupo particular de unidades experimentales. (Fisher., 1935).

El análisis de varianza ANOVA en una sola vía se unió a la prueba de rango múltiple de

«Duncan» y test de Tukey; se realizó con el software SPSS 11 para Windows, contrastando los datos entre especies y entre zonas (fototrópica, medio, geotrópica). Para éste análisis se determinò un $95 \%$ y $99 \%$ de confiabilidad. Se compararon los resultados de las variables mediante comparación de medias (prueba de "t") y regresión lineal. 


\section{RESULTADOS}

En la siguiente tabla se reporta el resumen ANOVA con el nivel de significancia de Fisher calculado de los índices de aceptación del 95\% y el 99\%, los datos de la prueba Duncan y el test Tukey, asì como los resultados de prueba de homogeneidad entre especies.

Tabla 7: Coeficientes de correlación entre las variables estudiadas (ANOVA) Correlation coefficients between variables studied

\begin{tabular}{|c|c|c|c|c|c|c|c|}
\hline Variable & $\begin{array}{l}\text { Prueba de } \\
\text { homogeneidad }\end{array}$ & $\begin{array}{l}\text { Signif } \\
95 \%\end{array}$ & Signif $99 \%$ & & & Duncan & Tukey \\
\hline pH & 2,63 & 0,67 & $(\mathrm{P}>0,05) \mathrm{NS}$ & 0,67 & $(\mathrm{P}<0,01) *$ & 0,404 & 0,101 \\
\hline MS & 0,354 & 0,07 & $(\mathrm{P}<0,05) *$ & 0,07 & $(\mathrm{P}<0,01) * *$ & 0,079 & 0,808 \\
\hline $\mathbf{C z}$ & 0,147 & 0,60 & $(\mathrm{P}>0,05) \mathrm{NS}$ & 0,60 & $(\mathrm{P}>0,01) \mathrm{NS}$ & 0,617 & 0,854 \\
\hline MO & 0,174 & 0,57 & $(\mathrm{P}>0,05) \mathrm{NS}$ & 0,57 & $(\mathrm{P}>0,01) \mathrm{NS}$ & 0,164 & 0,829 \\
\hline PC & 0,003 & 0,78 & $(\mathrm{P}>0,05) \mathrm{NS}$ & 0,78 & $(\mathrm{P}>0,01) \mathrm{NS}$ & 0,134 & 0,367 \\
\hline NNP & 0,739 & 0,50 & $(\mathrm{P}>0,05) \mathrm{NS}$ & 0,50 & $(\mathrm{P}>0,01) \mathrm{NS}$ & 0,522 & 0,772 \\
\hline PV & 0,939 & 0,74 & $(\mathrm{P}>0,05) \mathrm{NS}$ & 0,74 & $(\mathrm{P}>0,01) \mathrm{NS}$ & 0,252 & 0,594 \\
\hline PNV & 0,739 & 0,50 & $(\mathrm{P}>0,05) \mathrm{NS}$ & 0,50 & $(\mathrm{P}>0,01) \mathrm{NS}$ & 0,522 & 0,771 \\
\hline FDN & $\ldots \ldots \ldots$ & 0,86 & $(\mathrm{P}>0,05) \mathrm{NS}$ & 0,86 & $(\mathrm{P}>0,01) \mathrm{NS}$ & 0,329 & 0,983 \\
\hline FDA & $\ldots \ldots \ldots$ & 0,94 & $(\mathrm{P}>0,05) \mathrm{NS}$ & 0,94 & $(\mathrm{P}>0,01) \mathrm{NS}$ & 0,183 & 0,501 \\
\hline LDA & $\ldots \ldots \ldots$ & 0,94 & $(\mathrm{P}>0,05) \mathrm{NS}$ & 0,94 & $(\mathrm{P}>0,01) \mathrm{NS}$ & 0,91 & 0,992 \\
\hline Hc & ......... & 0,74 & $(\mathrm{P}>0,05) \mathrm{NS}$ & 0,74 & $(\mathrm{P}>0,01) \mathrm{NS}$ & 0,013 & 0,023 \\
\hline Dig & ......... & 0,43 & $(\mathrm{P}<0,05) * *$ & 0,43 & $(\mathrm{P}<0,01) * *$ & 0,45 & 0,082 \\
\hline PFT & .......... & 0,93 & $(\mathrm{P}>0,01) \mathrm{NS}$ & 0,97 & $(\mathrm{P}>0,01) \mathrm{NS}$ & 0,762 & 0,943 \\
\hline
\end{tabular}

No significativo (NS); $(* \mathrm{P}<0,05),(* * \mathrm{P}<0,01)$ 


\section{DISCUSIÒN}

Los resultados evidenciaron las posibilidades agronómicas y bromatológicas de las especies vegetales analizadas y evaluadas para su posible uso como alimento animal no convencional, principalmente para especies poligástricas según también la categoría y especie animal evaluada, las cuales dada las características morfológicas y funcionales de su tracto gastrointestinal las puede asimilar en forma eficiente estos forrajes.

\section{CONCLUSIONES}

Las variedades evaluadas se caracterizaron por una buena calidad bromatológica en general, el contenido de FAN se hallan dentro de los rangos promedio que puede tener un forraje que sea apto para ser usado como alimento animal, la presencia de algunos metabolitos secundarios pro-tóxicos o potencialmente causantes de afecciones digestivas no presupone que la biomasa presente mala calidad, se encuentran estos fitometabolitos en concentraciones moderadas; el índice de digestibilidad está de acuerdo con los valores promedio para los follajes de las especies leguminosas y otras familias botánicas en función de los análisis de la digestibilidad in vitro.

Cabe hacer el estudio desde el punto de vista de palatabilidad, sabor, textura, que son factores que inciden directamente en la aceptación del forraje como tal. 


\section{REFERENCIAS BIBLIOGRÁFICAS}

Araujo, J., Calvalcante, F., \& Da silva, L. (s.f.). ). Fenología y valor nutritivo de follajes de algunas especies forrajeras de la Caatinga1. Zootecnia Trop, 3(26), 231-234.

Baraona, R., \& Sánchez, S. (2005). Limitaciones físicas y químicas de la digestibilidad de pastos tropicales y estrategias para aumentarla. CORPOICA, 6(1), 69-82.

Botero, R., \& Russo, R. (s.f.). Utilización de arboles y arbustos fijadores de nitrógeno en sistemas sostenibles de producción animal en suelos ácidos tropicales. Recuperado 17 mayo, 2018, de http://www.fao.org/livestock/agap/frg/agrofor1/Botero8.htm

Carranza, L., Sánchez, L., \& Guzmán, R. (2003). Calidad y potencial forrajero de especies del bosque tropical caducidófilo de la sierra de Manantlan, México. (Ed. rev.). México, México: Colegio de Posgrados.

Cárdenas, E. (2003). Alternativas forrajeras para clima frío en Colombia. Recuperado de http://bdigital.unal.edu.co/29550/1/28071-99544-1-PB.pdf

García, A. (2003). Avances en investigación agropecuaria. Revista de investigación y difusión científica agropecuaria., 2(13), 25-39.

García, D., Medina, M., Moratinos, P., Cova, I., Torres, A., \& Santos, O. (2005).

Caracterización químico-nutricional de forrajes leguminosos y de otras familias botánicas empleando análisis descriptivo y multivariado. Instituto Nacional de Investigaciones Agrícolas (INIA), , 2(13), 25-39.

Santacoloma, L. (2011). Las dietas en las emisiones de metano durante el proceso de rumia en sistemas de producción bovina. Revista de Investigación Agraria y Ambiental RIAA, 2(1), 55-64. Recuperado de file://C:/Users/leonor.barreto/Downloads/document.pdf

Santacoloma, L., \& Granados, J. (2010). Evaluación del contenido de metabolitos secundarios en dos especies de plantas forrajeras encontradas en dos pisos térmicos de Colombia. Revista de Investigación Agraria y Ambiental - RIAA, 1(1), 31-35. Recuperado de http://hemeroteca.unad.edu.co/index.php/riaa/article/viewFile/890/891

Suárez, J., Carulla, J., \& Velásquez, J. (2008). Composición química y digestibilidad in vitro de algunas especies arbóreas establecidas en el piedemonte Amazónico. Zootecnia Tropical, 26(3), 55-64. Recuperado de Recuperado en 17 de julio de 2018, de http://www.scielo.org.ve/scielo.php?script=sci_arttext\&pid=S0798$72692008000300015 \& \operatorname{lng}=$ es\& tlng=es. 